\title{
Intermittent bilateral deep brain stimulation of the nucleus accumbens shell reduces intravenous methamphetamine intake and seeking in Wistar rats
}

\author{
Vinita Batra, PhD, ${ }^{1}$ Thanh Lam N. Tran, BS, ${ }^{2}$ Jessica Caputo, BS, ${ }^{2}$ Glenn F. Guerin, BS, ${ }^{2}$ \\ Nicholas E. Goeders, PhD, ${ }^{2}$ and Jessica Wilden, MD' \\ ${ }^{1}$ Department of Neurosurgery and ${ }^{2}$ Department of Pharmacology, Toxicology, \& Neuroscience, Louisiana State University Health \\ Sciences Center, Shreveport, Louisiana
}

OBJECTIVE There is increasing interest in neuromodulation for addiction. Methamphetamine abuse is a global health epidemic with no proven treatment. The objective of this study was to examine the effects of intermittent nucleus accumbens shell (AcbSh) deep brain stimulation (DBS) on operant methamphetamine intake and on methamphetamine seeking when stimulation is delivered in an environment different from that of drug use.

METHODS Eighteen rats were implanted with intravenous (IV) catheters and bilateral AcbSh electrodes and subsequently underwent daily sessions in 2-lever (active/methamphetamine and inactive/no reward) operant chambers to establish IV methamphetamine self-administration. After stable responding was achieved, 3 hours of DBS or sham treatment was administered (sham: $0 \mu \mathrm{A}, \mathrm{n}=8$; active: $200 \mu \mathrm{A}, \mathrm{n}=10$ ) in a separate nondrug environment prior to the daily operant sessions for 5 consecutive days. Immediately following each DBS/sham treatment, rats were placed in the operant chambers to examine the effects of remote stimulation on methamphetamine intake. After the 5 days of therapy were finished, rats reestablished a posttreatment baseline, followed by extinction training, abstinence, and 1 day of relapse testing to assess methamphetamine-seeking behavior.

RESULTS There was a decrease in total methamphetamine intake in rats receiving active DBS versus sham on Days 1 $(42 \%)$ and $2(44 \%)$. Methamphetamine administration returned to baseline levels following the cessation of DBS therapy. Compared with baseline drug responding, methamphetamine seeking was reduced (57\%) in the DBS group but not in the sham group.

CONCLUSIONS It is feasible to deliver noncontinuous DBS outside of the drug use environment with a resultant decrease in IV methamphetamine intake and seeking. The AcbSh is a neuroanatomical substrate for psychostimulant reinforcement and may be a target for intermittent neuromodulatory therapies that could be administered during brief periods of sobriety.

https://thejns.org/doi/abs/10.3171/2016.4.JNS152524

KEY WORDS deep brain stimulation; methamphetamine; intravenous self-administration; substance addiction; neuromodulation; relapse

$\mathrm{M}$ ETHAMPHETAMINE abuse is an epidemic health problem involving an estimated 25 million users globally. ${ }^{44}$ Young people who abuse methamphetamine have higher morbidity and mortality rates than their peers ${ }^{39}$ and may suffer significant long-term cardiovascular disease, psychosis, and cognitive and motor impairments that mimic those observed in Parkinson's disease..$^{21}$ This excess mortality, along with health care expenses, law enforcement costs, property loss, and reduced productivity, contribute to the considerable economic burden of methamphetamine abuse and dependence, which is approximately $\$ 23$ billion per year in the US. ${ }^{10}$ Unlike many drugs of abuse, there are no proven psychosocial or pharmacological treatments for methamphetamine de-

ABBREVIATIONS Acb = nucleus accumbens; AcbSh = nucleus accumbens shell; $D B S=$ beep brain stimulation; $D_{1 R}=D_{1}$-receptor; FR1 = fixed ratio of one; GABA = $\gamma$-aminobutyric acid; HFS = high-frequency stimulation; IV = intravenous; LFS = low-frequency stimulation; MSN = medium spiny neuron; PFC = prefrontal cortex; PSR = Pavlovian spontaneous recovery; TMS = transcranial magnetic stimulation; VTA = ventral tegmental area.

SUBMITTED October 29, 2015. ACCEPTED April 12, 2016.

INCLUDE WHEN CITING Published online July 8, 2016; DOI: 10.3171/2016.4.JNS152524. 
pendence, ${ }^{10}$ and rates of recidivism are extremely high. ${ }^{6}$ There is thus a substantial need to elucidate the underlying environmental and neurobiological factors that contribute to methamphetamine dependence and to develop novel therapeutic strategies.

To establish a neurosurgical treatment for methamphetamine dependence, candidate brain locations must be identified that regulate methamphetamine intake and seeking in an animal model. Methamphetamine is a psychostimulant that crosses the blood-brain barrier easily, producing an intense and prolonged euphoria due to an acute increase in synaptic monoamines, including dopamine, serotonin, and norepinephrine..$^{12}$ As with most drugs of abuse, methamphetamine's mechanisms produce acute reinforcing actions at least partially through the mesocorticolimbic system, which includes dopaminergic projections from the ventral tegmental area (VTA) to the nucleus accumbens (Acb), medial prefrontal cortex (PFC), and amygdala. ${ }^{13,35,38,45,52}$ Specifically, the medioventral subterritory of the Acb, or the Acb "shell" (AcbSh), is one critical region for the self-administration of psychostimulants $\mathrm{s}^{31,48,55}$ and shows compromised function after withdrawal from chronic methamphetamine. ${ }^{7,35}$

There is compelling, albeit limited, preclinical evidence that the Acb can be locally altered with medication or stimulation to attenuate relapse to psychostimulants. Context-induced, and cocaine-priming-induced, reinstatement of cocaine-seeking behavior has been reduced by intra-Acb infusions of $\gamma$-aminobutyric acid (GABA) agonists ${ }^{19}$ and of a $D_{1}$-receptor $\left(D_{1 R}\right)$ antagonist, ${ }^{2}$ respectively, when these therapies were administered just prior to exposure to the drug use environment. Similarly, contextand drug-induced reinstatement of methamphetamine seeking has been prevented by intraaccumbens infusions of GABA agonists. ${ }^{49}$ Optogenetic stimulation of the Acb has recently been used to decrease cocaine self-administration, ${ }^{5}$ cocaine priming- and cue-induced cocaine seeking, ${ }^{54}$ and cocaine context-associated memory. ${ }^{62}$ More clinically relevant, deep brain stimulation (DBS) of the bilateral accumbens has been shown to decrease cocaine priming- and cue-induced reinstatement of cocaine seeking ${ }^{58,59}$ although the effects on cue-induced seeking were not drug specific and also affected relapse to sucrose. ${ }^{24}$

To our knowledge, there have been no preclinical studies regarding Acb DBS for methamphetamine abuse. This lack of studies may be a by-product of the implicit difficulty in translating research findings into human therapy. Methamphetamine abusers may have psychiatric comorbidities, decline in cognitive function (temporary or permanent), poor psychosocial support, significant infection risk factors (e.g., intravenous [IV] drug use, poor hygiene), and noncompliance; ${ }^{10,41}$ these are all factors that would currently rule out the use of an indwelling DBS device in common clinical practice. One weakness of some existing preclinical studies examining cocaine is the imitation of the classic but impractical DBS paradigm. Multiple studies have examined DBS effects when the therapy is delivered continuously during psychostimulant self-administration in the drug use environment. ${ }^{24,58,59}$

A noninvasive neuromodulatory therapy that is delivered remotely from the drug use setting while still main- taining efficacy would be ideal for psychostimulant abusers. While the development and implementation of such an ideal treatment is a seemingly unattainable goal, recent human case reports suggest that transcranial magnetic stimulation (TMS), a type of noninvasive brain stimulation delivered in 5-10 15-minute clinic visits, may decrease cocaine craving as well as cocaine use in the short term. ${ }^{20,56}$ Although clearly an area deserving of further study, reverse translation is difficult because technical limitations and anatomy prevent the use of TMS in awake, behaving rodents..$^{15}$ Nonetheless, the first step toward preclinical development of novel neuromodulatory therapies is to demonstrate that noncontinuous repeated brain stimulation using implanted intracranial electrodes in rodents can affect drug-related behaviors that occur in a different environment and at a different time than that of the stimulation. Augmenting the preexisting, albeit limited, data on cocaine, ${ }^{18,26,36}$ this study describes the effects of noncontinuous repeated brain stimulation of the AcbSh on the acute self-administration of IV methamphetamine and on remote methamphetamine seeking.

\section{Methods}

For clarity, an overview of the experimental timeline is shown in Fig. 1.

\section{Animals}

Experimentally naïve adult male Wistar rats weighing $300-350 \mathrm{~g}$ were used in the present study. The rats were single-housed on arrival at the lab and were maintained on a 12-hour reverse light-dark cycle (lights off at 6:00 AM). The rats were maintained at $85 \%-90 \%$ of their free-feeding body weights and weighed daily, Monday through Friday, to minimize any fluctuations. Prior experience in our lab has shown that rats more readily self-administer psychomotor stimulants when maintained within this weight range and maintain healthier, longer lives compared with rats allowed to feed ad libitum. Water was freely available throughout the experiment, except for periods during which the animals were in the test chambers. The rats used in this study were maintained in facilities fully accredited by the Association for the Assessment and Accreditation of Laboratory Animal Care. All research protocols were approved by the Institutional Animal Care and Use Committee and were in accordance with guidelines of the Institutional Care and Use Committee of the National Institute on Drug Abuse, NIH, and the Guide for the Care and Use of Laboratory Animals (National Research Council, 1996).

\section{Implantation of Jugular Catheters}

Anesthesia for all rats having the IV catheter procedure consisted of pentobarbital (20-50 mg/kg, intraperitoneally) with methyl atropine nitrate pretreatment (10 $\mathrm{mg} / \mathrm{kg}$, intraperitoneally). Each rat was then implanted with a chronic indwelling jugular catheter. The catheter (0.012 inches inner diameter $\times 0.025$ inches outer diameter, silicone tubing) was inserted into the right posterior facial vein and pushed down into the jugular vein until it terminated outside the right atrium. The catheter was 


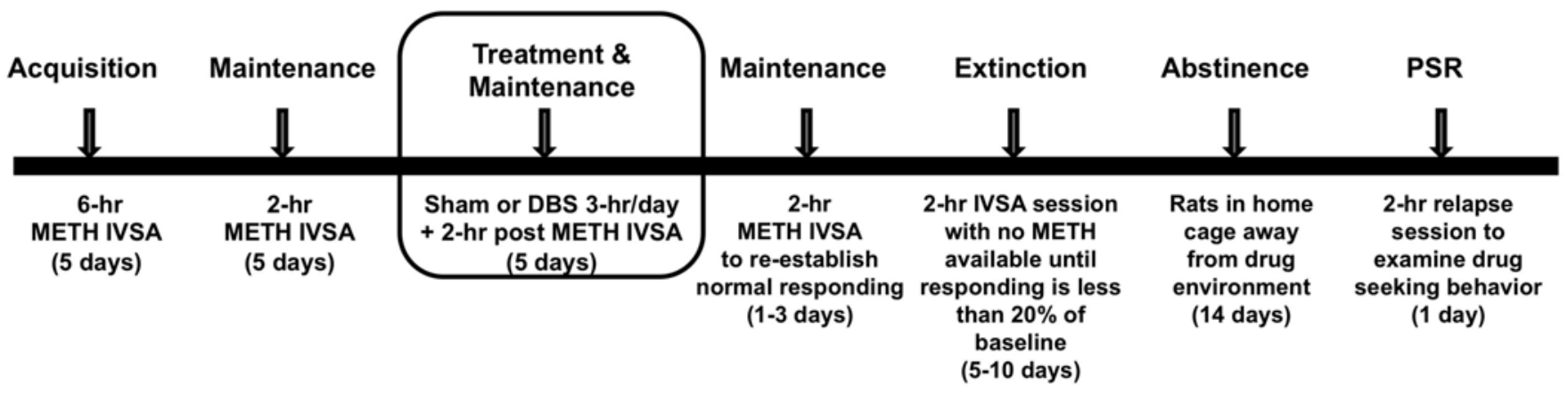

FIG. 1. Experimental timeline from initial IV self-administration (IVSA) training until relapse testing during the PSR session. METH $=$ methamphetamine.

anchored to the vein and continued subcutaneously to the back where it exited just posterior to the scapulae through a Marlex mesh/dental acrylic/22-gauge guide cannula (Plastic Products) assembly that was implanted under the skin for attachment of a leash. The patency of the catheters was tested at least once each week. If blood could be obtained via the catheter, then it was judged to be patent. If not, then the rat was injected via the catheter with methohexital sodium (1.5 mg, IV). An immediate light anesthesia indicated that the catheter was functional. If a catheter became blocked, reimplantation was attempted, and if unsuccessful, the rat was removed from the study.

\section{Implantation of Intracranial Electrodes}

Once animals recovered from IV catheter implantation (approximately 1 week), each rat underwent isoflurane induction of general anesthesia followed by stereotactic implantation of bilateral twisted electrode pairs (platinum microwires in Teflon, inner diameters $0.125 \mathrm{~mm}$ each, Plastics One, Inc.) into the AcbSh (+ $1.8 \mathrm{~mm}$ anterior from bregma, $\pm 2.4 \mathrm{~mm}$ from the midline, and $8.5-\mathrm{mm}$ ventral, $10^{\circ}$ angle). Each electrode terminated in 2 female sockets, which were connected to a dual-channel electrode pedestal and fixed into place with cranioplastic material (OrthoJet, Lang Dental Manufacturing Co.) layered over 4 stainless steel screws fastened to the skull. Protective caps were placed over the head of the electrode pedestals to prevent infections or the formation of obstructions and remained in place except during testing. After surgery, the rats remained individually housed and were allowed to recover for approximately 4-5 days before testing was initiated.

\section{Operant IV Methamphetamine Self-Administration}

Standard plastic and stainless-steel operant conditioning chambers contained within sound-attenuating enclosures (Med-Associates, Inc.) were used to run the behavioral experiments. Each experimental chamber was equipped with 2 response levers mounted on 1 wall of the chamber with a stimulus light located above each lever. One was the "active" lever that resulted in drug delivery from an external infusion syringe-pump, while the other was the "inactive" lever. The enclosures contained an exhaust fan that supplied ventilation and white noise to mask extraneous sounds. An IBM-compatible personal computer and interface system was used to program the procedures and collect the experimental data. A stainless-steel spring leash (Plastic Products) was attached to the guide cannula assembly on the rodent's back and to a leakproof fluid swivel suspended above the chamber during experimental sessions. Methamphetamine was obtained from the National Institute on Drug Abuse. The drug was delivered intravenously to the animals as an infusion of 0.05 $\mathrm{mg} / \mathrm{kg} /$ infusion in $100 \mu \mathrm{l}$ of $0.9 \% \mathrm{NaCl}$ delivered over 2.8 seconds.

Rats were trained to respond under a fixed ratio of one (FR1) schedule of methamphetamine self-administration, meaning that the rats received 1 methamphetamine reward for each active lever press. During an active session, a stimulus light located directly above the "active" response lever was illuminated to indicate the availability of drug. Depression of the active lever resulted in drug infusion followed by concurrent darkening of the stimulus light for a 30-second timeout. A cue house light was briefly activated for 5 seconds during the drug infusion phase. This cue served as a secondary, or conditioned, reinforcer. This was followed by a brief timeout period after infusion, during which responses on the active lever were counted but had no scheduled consequences. Depressions of the "inactive" lever were also recorded but had no scheduled consequences.

The rats were not acclimated to the operant chamber before commencement of the experiment and did not receive any prior operant training. Rats learned spontaneously to press the lever for an IV infusion of methamphetamine in daily 6-hour sessions during the first week of exposure. This phase of escalating responding was defined as the "acquisition" of operant drug self-administration. Previous experiments indicated that this initial prolonged and "easy" access results in the majority of rodents acquiring significant drug-taking behavior in less than 1 week. ${ }^{3}$ During the 2 nd and $3 \mathrm{rd}$ weeks of training, rats underwent 2-hour daily sessions at approximately the same time Monday through Friday, again on an FR1 schedule of reinforcement culminating in the rats developing a baseline intake termed "maintenance." Maintenance was operationally defined as stable, intense responding for which the total number of methamphetamine infusions across each session varied less than approximately $10 \%$ for 3 consecutive sessions and the cumulative number of infusions across the 1st 30 minutes was greater than, or equal to, the cumulative number of infusions during the 2nd 30 minutes. This criterion ensured that the rats developed a 
drug-loading pattern that indicated addictive behavior ${ }^{1}$ and not simply casual use by the time treatment was initiated. ${ }^{3}$ Once maintenance had been established, rats were counterbalanced based on total baseline 2-hour IV methamphetamine infusions and divided in 2 groups, active stimulation (DBS) and sham stimulation (sham).

\section{Deep Brain Stimulation}

DBS and sham therapy were conducted in a distinct environment from that of operant drug self-administration. The rodent DBS system consisted of 8 Plexiglas boxes (12 $\times 18 \times 18$ inches [width $\times$ height $\times$ depth]). Each chamber was equipped with an electrical swivel, or commutator (Plastics One), embedded in a panel that fit over the top of the testing boxes. Commutators facilitated the electrical connection between the rodent head cap and the stimulation system via 2 animal-proof stainless steel 2-channel cables, which attached proximally to each implanted electrode pedestal and distally to the electrical swivel (Plastics One) to allow free movement within the cage. A customized stimulation system (Tucker Davis Technologies) that maintained constant current stimulation throughout the therapy sessions was used. Prior to each DBS session, the electrochemical impedance of the electrode was measured to ensure proper functioning of the system and adequate current delivery. Prior to active therapy, rats underwent 1 or 2 mock DBS sessions in which they were placed in the DBS boxes and tethered to the DBS system via head cables in the absence of active therapy. This session was conducted to eliminate nonspecific behavioral effects and to allow habituation to the new environment. Following the mock testing, active or sham stimulation was administered to each rat for 3 hours a day. At the end of the 3-hour stimulation session, rats were immediately transferred into the operant chambers for a 2-hour methamphetamine selfadministration session to determine DBS effects on acute drug intake. The stimulus parameters were based on prior work: ${ }^{59,63}$ biphasic, rectangular pulses with no interphasic delay; pulse frequency $130 \mathrm{~Hz}$; pulse width $60 \mathrm{msec}$; and current intensity of $200 \mu \mathrm{A}$. Rats received 5 consecutive days of active or sham stimulation.

\section{Examination of Methamphetamine Seeking}

In early studies of conditioned learning, Pavlov noted that, after a series of extinction trials sufficient to eliminate responding, a brief period of removal from the testing chamber would reinstate responding. He termed this phenomenon spontaneous recovery and defined it as a recovery of responding, in the absence of the previously trained reward, which is observed after a period of rest after extinction. ${ }^{50}$ This Pavlovian spontaneous recovery (PSR) is a measure of context- and cue-induced drug seeking and serves as one possible surrogate for drug craving and relapse. . $7,50^{27}$

After the 5 days of DBS or sham therapy, rats continued 2-hour daily operant training to reestablish stable baseline responding on an FR1 schedule for IV methamphetamine. Rats then underwent extinction training, also on an FR1 schedule, during which depression of the active lever resulted in no scheduled methamphetamine delivery, but presentation of the stimulus light and cue light occurred the same as before. Rats underwent 2-hour daily operant extinction sessions until responding levels were less than $20 \%$ of baseline. Once the criterion was met for extinction, rats were placed in the home cage environment for 14 consecutive days with no access to methamphetamine, the operant chambers, or associated cues. Rats were weighed and handled daily during abstinence.

On the 15th day of abstinence, rats underwent PSR testing as a measure of methamphetamine seeking. Rats were placed in the operant chambers and the stimulus light above the active lever was illuminated. Depression of the active lever resulted in the presentation of the usual conditioned cues (e.g., activation of the house cue light for 5 seconds followed by darkening of the stimulus light for 30 seconds in the chamber) but did not result in any IV methamphetamine delivery. Depression of the inactive lever resulted in no scheduled consequences. Rats underwent a single 2-hour PSR session.

\section{Food Self-Administration}

A separate cohort of rats was likewise trained to selfadminister palatable food pellets on a "fixed ratio of 4" schedule during which 4 lever presses resulted in the presentation of a food reward. The increased work requirement was used given the known high response rate for food pellets noted previously in prior experiments. All the methods described above were the same except that in place of IV methamphetamine infusion, rats received a food pellet and were not tethered to an IV leash. After stable food responding was achieved, rats followed the DBS protocol as described.

\section{Histological and Data Analysis}

At the termination of the experiment, rats were anesthetized using pentobarbital, and an anodal current of 50 $\mu \mathrm{A}$ was passed through the brain for 120 seconds to burn the location of the tip of each electrode. Immediately after lesioning, rats were decapitated. The brains were removed en bloc and stored at $-20^{\circ} \mathrm{C}$. Subsequently, the frozen brains were equilibrated at $15^{\circ} \mathrm{C}$ in a cryostat microtome and sliced into 40- $\mu \mathrm{m}$ sections (Fig. 2). Slices were then examined for verification of the DBS site by using the rat brain atlas of Paxinos et al. ${ }^{47}$ Only those rats in which accurate placement of electrodes was confirmed were included in the final data analysis. Analyses for the maintenance of operant data consisted of a mixed ANOVA, with a between-subject variable of treatment group and a repeated measure of IV methamphetamine infusions per day. Data analysis for PSR consisted of t-tests to compare baseline IV methamphetamine intake to number of infusions during the drug-seeking session. The $\mathrm{p}$ value of significance was set at $\leq 0.05$ for all analyses.

\section{Results}

\section{Between-Group Analysis}

Both DBS $(n=10)$ and sham $(n=8)$ groups had similar baseline levels of methamphetamine infusions, with the 


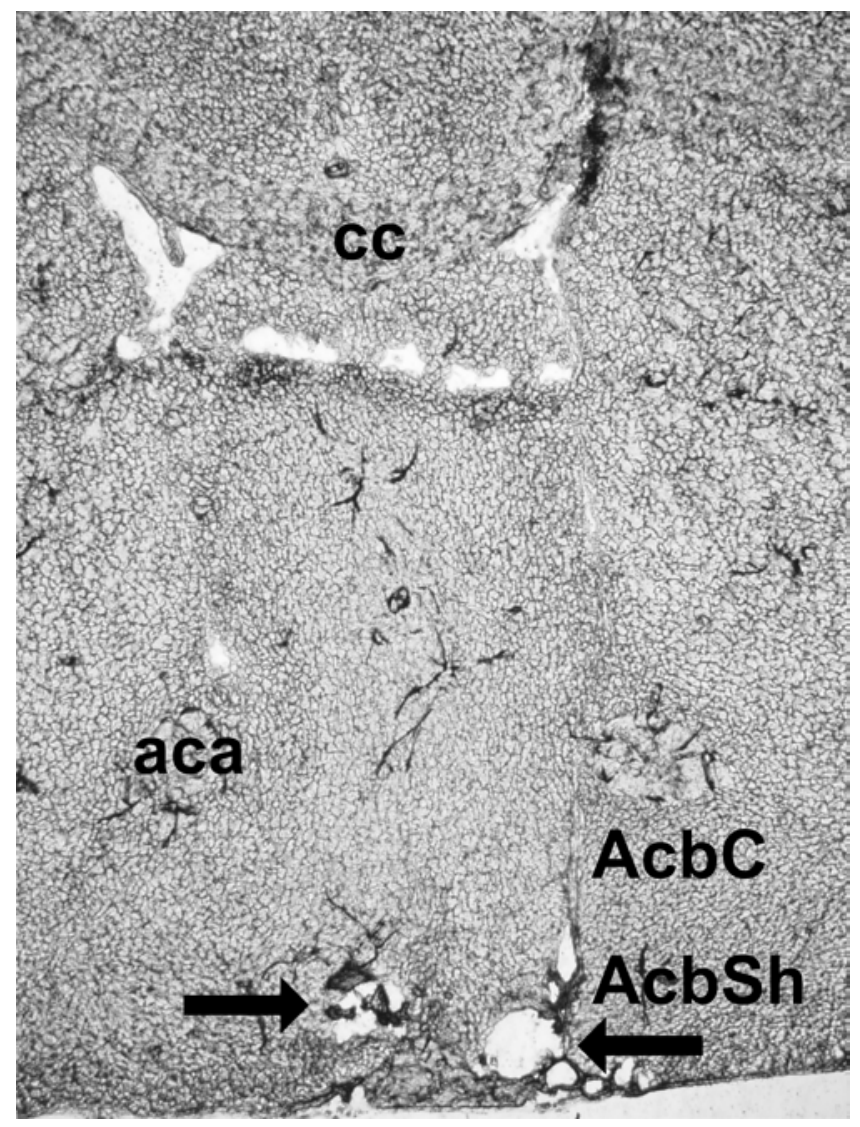

FIG. 2. Unstained brain section at a magnification of $\times 1.5$ demonstrating typical bilateral electrode placements (arrows) in the ventromedial AcbSh. aca $=$ anterior commissure; $\mathrm{AcbC}=$ nucleus accumbens core; $c c$ = corpus callosum.

average number of active responses resulting in approximately 88 methamphetamine infusions over a 2-hour session (Fig. 3 "Base"). The overall analysis indicated that there was a significant treatment group $x$ day interaction $(F(6,96)=2.22 ; p=0.047)$. Deconstructing the interaction term by holding the day constant (examining the effects of treatment group on each individual day) indicated that active DBS of the AcbSh reduced subsequent IV methamphetamine self-administration during the operant sessions on Days 1 (42\% decrease; $p<0.05$; Fig. 3 ) and 2 (44\% decrease; $\mathrm{p}<0.05$; Fig. 3). Posttreatment, rats in both groups returned to baseline levels of responding that were not significantly different between groups (Fig. 3 "Post").

\section{Within-Group Analysis}

One-way ANOVA indicated that there was a main effect of day $(F(6,54)=3.46, p=0.01)$ in the DBS group. Tukey post hoc testing indicated that DBS of the AcbSh reduced IV methamphetamine self-administration compared with baseline responding (Fig. 4 "Base") during the operant sessions on Days 2 (40\% decrease; $p=0.023$; Fig. $4)$ and $5(38 \%$ decrease; $p=0.036$; Fig. 4$)$ with a trend toward decreasing responding on Day 1 (34\% decrease; $\mathrm{p}=0.1$; Fig. 4). Posttreatment, rats returned to baseline levels of responding (Fig. 4 "Base, Post"). One-way ANO-
VA indicated that there was no difference between baseline responding and any treatment day in the sham group $(\mathrm{F}(6,42)=0.83 ; \mathrm{p}=0.55)$. Posttreatment levels of responding were similar to baseline responding.

\section{Daily, Intermittent AcbSh DBS and Remote Methamphetamine Seeking}

Both DBS $(n=10)$ and sham $(n=8)$ rats demonstrated methamphetamine seeking during the PSR test by pressing the active lever for an infusion even in the absence of a tangible IV methamphetamine reward, self-administering a total of 38 and 62 nondrug infusions, respectively. Methamphetamine seeking between treatment groups was not significantly different (2-sample t-test, $\mathrm{p}=0.11$; Fig. 5), although there was a mild trend toward the DBS group having a lower infusion rate (38\% decrease). Within-group comparisons revealed that rats that had received DBS therapy during maintenance had significantly lower nondrug infusion administration compared with baseline responding during PSR testing (paired t-test, 57\% decrease; $\mathrm{p}<$ 0.01 ; Fig. 5). Rats that had received sham therapy during maintenance also had lower nondrug infusion administration than baseline during PSR testing but this result did not reach significance (paired t-test, 30\% decrease; $\mathrm{p}=$ 0.11; Fig. 5).

\section{Daily, Intermittent AcbSh DBS and Acute Food Self- Administration}

Two-way repeated measures ANOVA indicated no difference between sham and DBS rats during the 5 treatment days in regard to food pellet self-administration $(\mathrm{F}(1,40)=$ $0.43 ; p=0.53$; Fig. 6 ).

\section{Discussion}

This study found that 5 days of intermittent bilateral DBS of the AcbSh reduces acute IV methamphetamine intake and subsequent methamphetamine seeking. This is the first time that DBS therapy temporally and spatially separate from the drug use environment has decreased methamphetamine abuse and relapse in Wistar rats. These suppressive effects were selective for methamphetamine and did not affect the acute self-administration of food. Our findings have positive implications that addiction behaviors may be altered by noncontinuous neuromodulatory therapies delivered repeatedly in a sober environment. Notably, rats that received AcbSh DBS only during the maintenance phase of methamphetamine use demonstrated reduced relapse 4-5 weeks later after a period of detoxification and abstinence. This suggests that DBS of the AcbSh may exert both acute and chronic effects on methamphetamine-related behaviors and may modulate different stages of addiction $^{32}$ after a single treatment course.

\section{Pathophysiology of Methamphetamine Abuse}

To further understand our results, one must first understand the neurological effects of methamphetamine. Methamphetamine causes dysfunction of the mesocorticolimbic system, including the dopaminergic neurons in the ventral mesencephalon, which project to the Acb, 


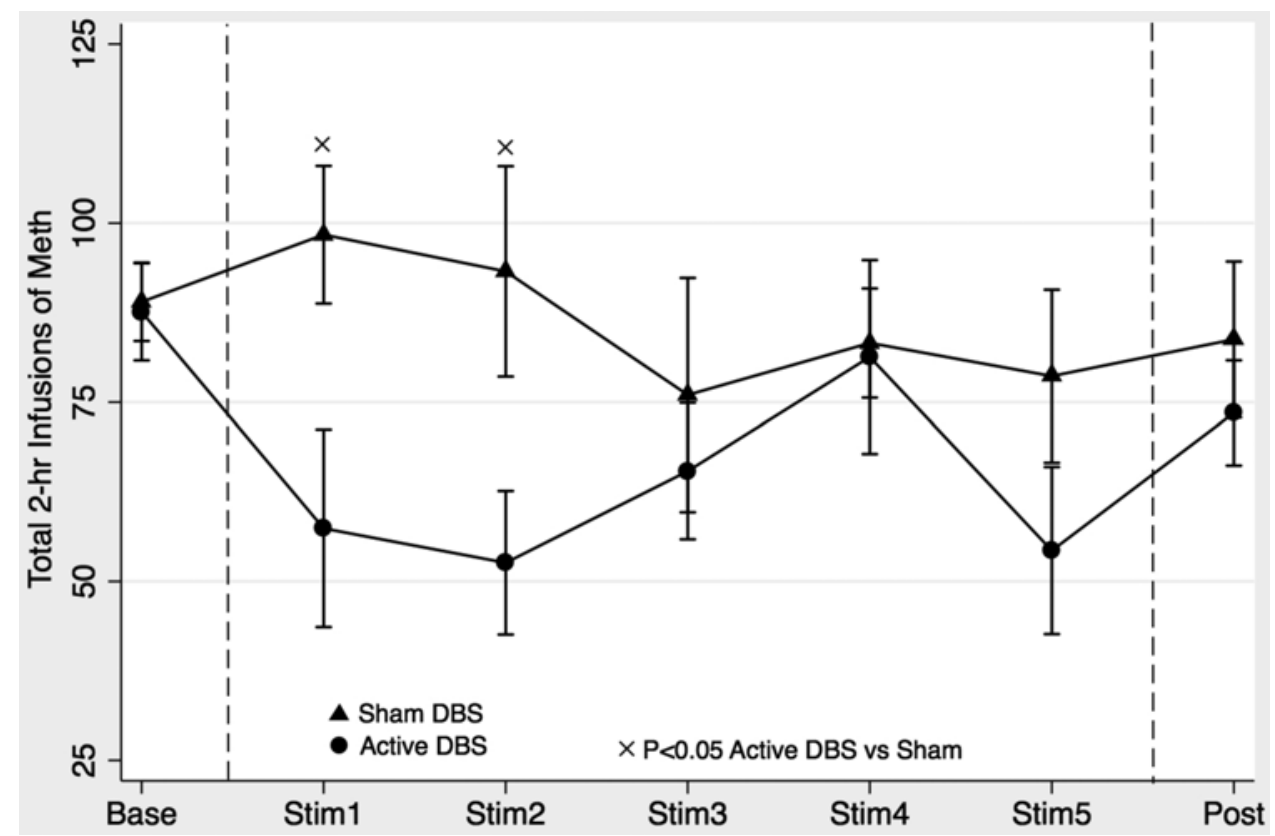

FIG. 3. Total self-administered infusions of IV methamphetamine over each daily 2-hour operant session. Data are means \pm standard errors. Bilateral preoperant DBS significantly reduced the total number of IV methamphetamine infusions on treatment Days 1 and 2. $p<0.05$ compared with sham group. The average number of active responses resulting in an infusion of methamphetamine returned to baseline levels after daily treatment ended. Base = baseline; Post = posttreatment; Stim = stimulation.

the frontal cortex, and the amygdala to facilitate reward. ${ }^{13,35,38,45,52}$ Methamphetamine's chemical structure is similar to that of monoamines, including dopamine and serotonin ${ }^{10,12}$ and its acute mechanism of action is 2 -fold: 1) methamphetamine binds to transmembrane monoamine reuptake transporters and reverses their function, causing an increase in the release of monoamines into the cytosol and the synapse; and 2) methamphetamine inhibits monoamine oxidase, decreasing the natural breakdown of monoamines with resulting buildup of these compounds in the synapse..$^{10,12}$ These actions occur largely at the synapses of dopamine axonal terminals in the Acb and are

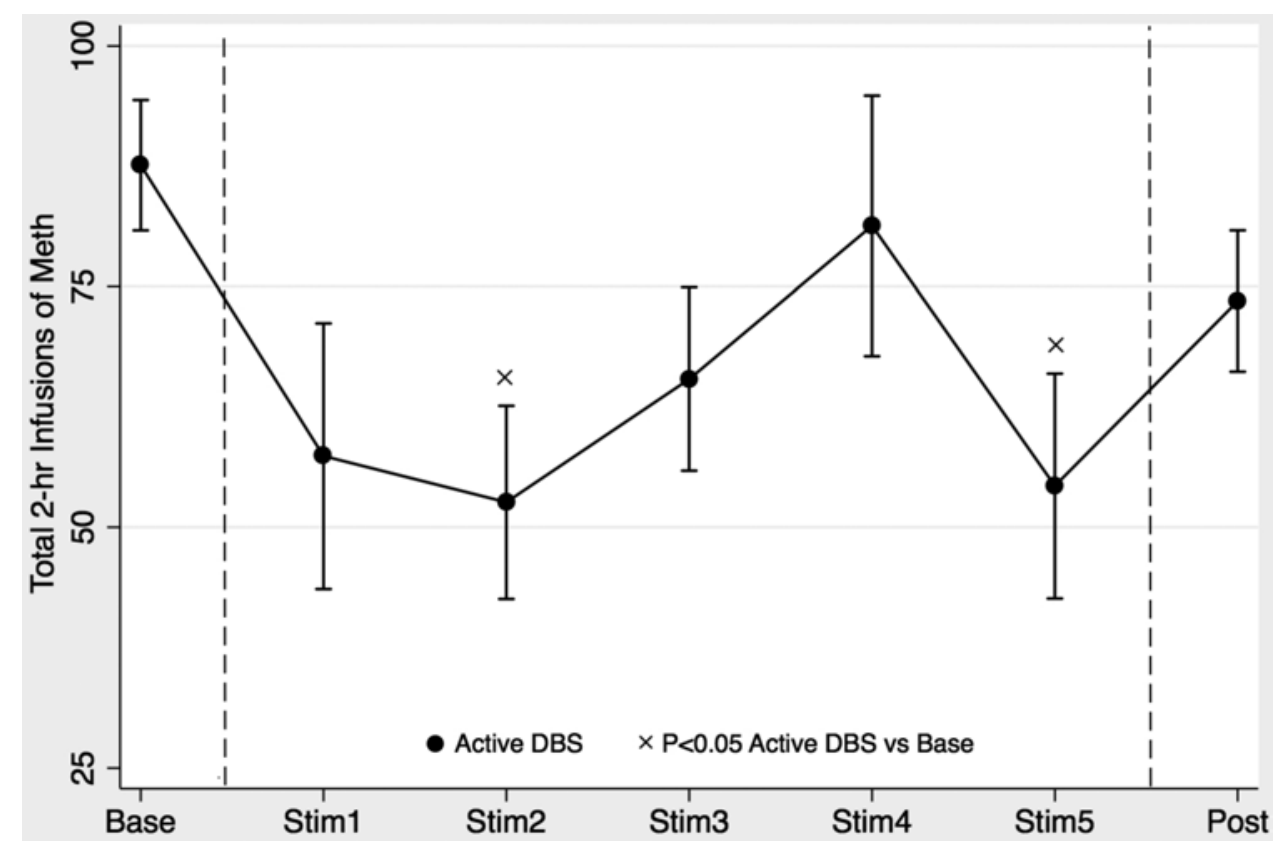

FIG. 4. Total self-administered infusions of IV methamphetamine over each daily 2-hour operant session. Data are given as means \pm standard errors. Bilateral preoperant DBS significantly reduced the total number of methamphetamine infusions on treatment Days 2 and 5 compared with baseline responding. $p<0.05$ compared with baseline. Responding returned to baseline levels after daily treatment ended. 


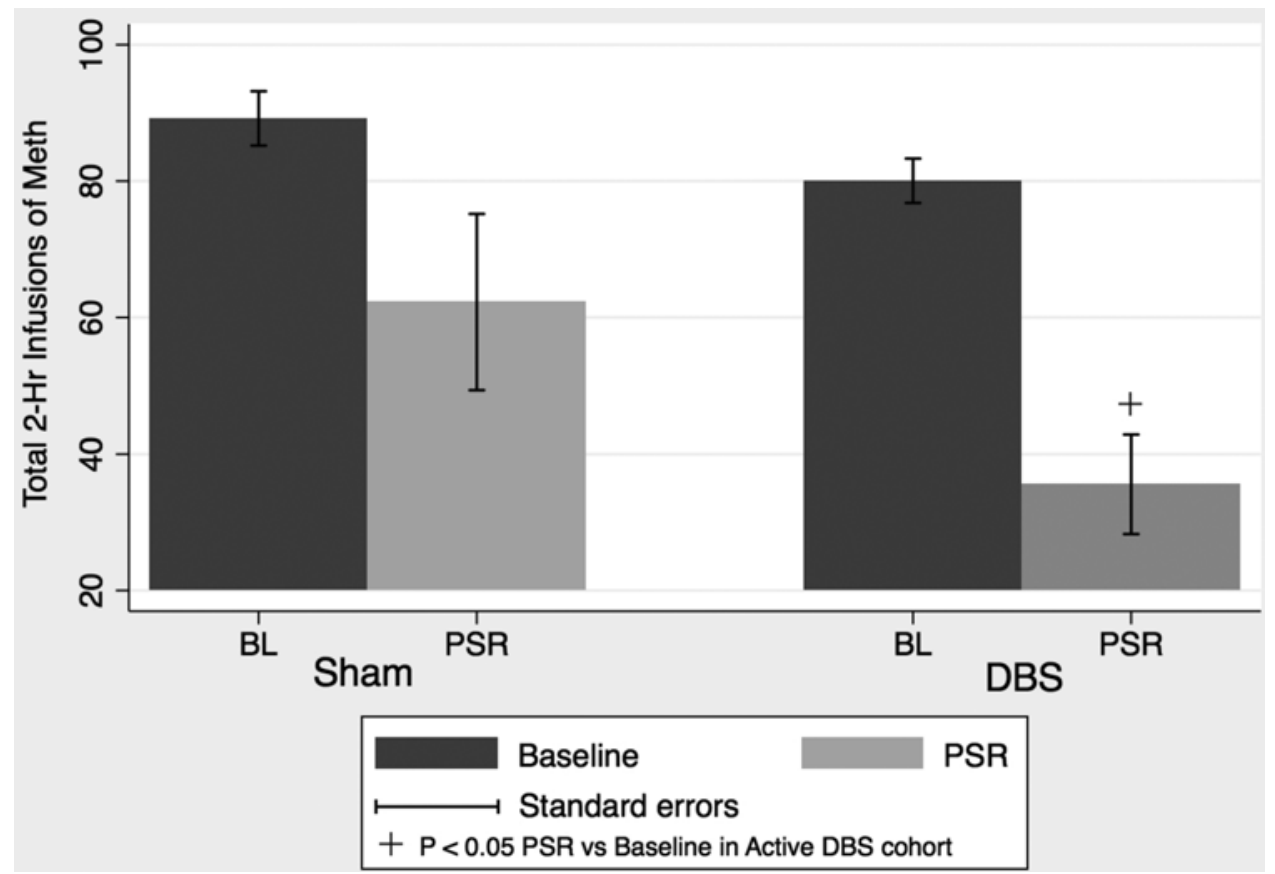

FIG. 5. Total self-administered infusions of IV methamphetamine over the 2-hour relapse session. Data are means \pm standard errors. Bilateral preoperant DBS treatment significantly reduced methamphetamine seeking during PSR compared with baseline responding. Bilateral preoperant sham treatment did not result in a significant difference between baseline and PSR responding. Sham and DBS treatment groups did not differ during baseline or PSR. $p<0.05$ compared with baseline. $B L=$ baseline.

responsible for the intense euphoria that addicted patients experience when using methamphetamine..$^{10,12}$

While acute administration of psychostimulants enhances dopaminergic function in the Acb, ${ }^{13,38}$ chronic exposure reduces baseline dopaminergic transmission and stressand drug-induced dopamine responsiveness in the $\mathrm{Acb}^{7,35}$ and decreases striatal molecular markers of dopamine

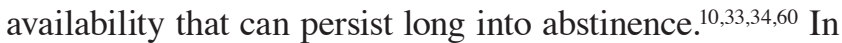
a selectively bred model of methamphetamine-preferring rats compared with control animals, basal dopamine content in the Acb is significantly decreased, dopamine sensitivity to drug administration is enhanced in the medial PFC, and monoamine receptors/transporters are altered in both regions. ${ }^{38}$ Taken together, this evidence suggests that

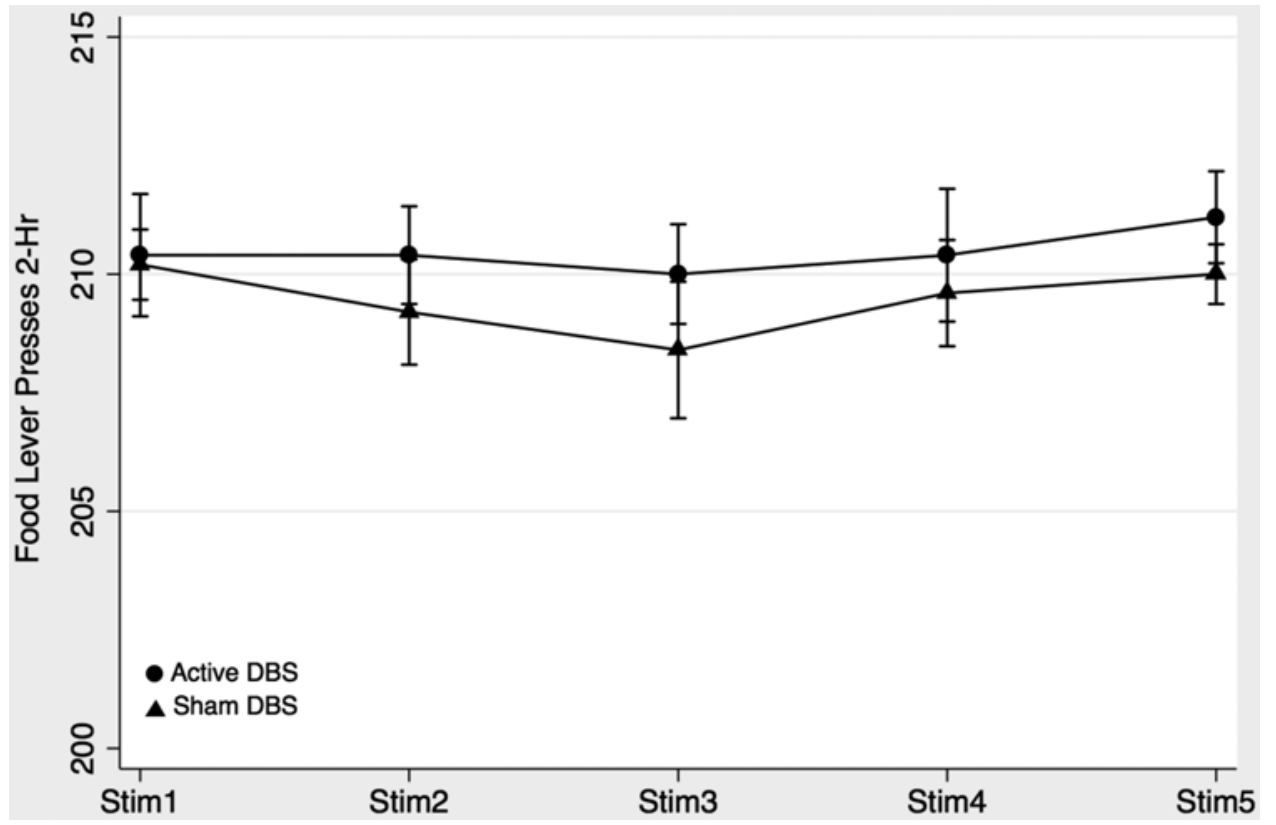

FIG. 6. Total self-administered food pellets over each daily 2-hour operant session. Data are means \pm standard errors. Bilateral preoperant DBS did not affect responding for food on any treatment day compared with sham. 
chronic methamphetamine abuse may lead to a widespread disease of decreased dopaminergic tone, which impacts the function of the mesocorticolimbic system in a manner similar to the way Parkinson's disease impacts the nigrostriatal system. Furthermore, methamphetamine may also damage the nigrostriatal pathways, as indicated by the observation of neurocognitive and parkinsonian motor deficits in both animal models $22,30,35,51$ and a subset of human abusers. ${ }^{8,10,61}$ In support of this assertion, a recent study demonstrated that a history of heavy methamphetamine self-administration in rodents causes a progressive degeneration of nigrostriatal projections as well as lateral mesolimbic projections that mimic the neuronal loss noted in Parkinson's disease. ${ }^{33}$

In addition to its neurochemical and neurodegenerative effects, psychostimulant abuse may also lead to longlasting alterations in neuronal communication. PFC, glutamatergic, and VTA dopaminergic inputs onto local $\mathrm{D}_{1} /$ $\mathrm{D}_{2}$ receptors on Acb medium spiny neurons (MSNs) may play a critical role in psychostimulant consumption and relapse. The Acb is located within the ventral striatum, which like the dorsal striatum, contains $\mathrm{D}_{1 \mathrm{R}}$-containing GABAergic MSNs that project directly to the midbrain (e.g., a "direct" pathway) and $\mathrm{D}_{1 \mathrm{R}^{-}}$and $\mathrm{D}_{2 \mathrm{R}}$-containing GABAergic MSNs that project to the midbrain indirectly via the ventral pallidum and subthalamic nucleus (e.g., an "indirect" pathway). ${ }^{5,23}$ Chronic psychostimulant addiction is likely a disease of dopamine deficiency ${ }^{7,35}$ coupled to altered neuronal plasticity ${ }^{14,46}$ that results in hypoactivity of the indirect Acb-brainstem pathway during the initial/ ongoing maintenance of drug use $e^{5,37}$ and hyperactivity of the direct Acb-brainstem pathway in withdrawal after chronic use $\mathrm{s}^{5,14,46}$ (Fig. 7). Although the behavioral roles are far from clear, a hypoactive $D_{2 R}$ MSN indirect pathway may contribute to ongoing compulsive drug intake ${ }^{5}$ and enhanced drug memory ${ }^{62}$ and a hyperactive $\mathrm{D}_{1} \mathrm{MSN}$ direct pathway may contribute to drug craving and subse- quent relapse $\mathrm{e}^{11}$ because a dose of cocaine or methamphetamine temporarily relieves this neuronal overactivity. ${ }^{29}$ While we acknowledge that this description of addiction pathophysiology is a gross oversimplification, ${ }^{53}$ it nonetheless provides a framework upon which to understand and refine stimulation effects.

\section{Acute Effects of DBS}

This study demonstrated an acute decrease in consumption of IV methamphetamine after daily, 3-hour AcbSh high-frequency stimulation (HFS) in the immediate predrug period. Only 1 other study has examined Acb DBS treatment prior to operant self-administration of a psychostimulant, specifically cocaine, and this study did not find a DBS-induced decrease in drug intake. ${ }^{26}$ However, this discrepancy could be due to the fact that we delivered DBS for a longer period of time ( 3 hours vs 30 minutes) and repeatedly for several consecutive days as opposed to a single session. It is also important to note that repeated exposure to cocaine versus amphetamine leads to distinct neurobiological changes in the Acb region ${ }^{29}$ so caution is advised in assuming a 1:1 comparison between our study and studies of cocaine. The mechanisms of DBS are far from clear, but preclinical ${ }^{9,28,42,57}$ and clinical evidence suggest that the therapeutic effects of DBS in Parkinson's disease $^{64}$ and obsessive-compulsive disorder ${ }^{16}$ may be partly achieved by increasing dopamine content and upregulating dopamine-related enzymes in the CNS. Given that chronic methamphetamine self-administration may lead to a dopamine deficit at the $\mathrm{D}_{2 \mathrm{R}} \mathrm{MSN}$ during ongoing drug use (Fig. 7), the acute effects of DBS observed in our study could have been due to short-lived increases in dopamine transmission at these receptors, which may have led to decreased methamphetamine intake.

Admittedly, the DBS effects we observed on IV meth-
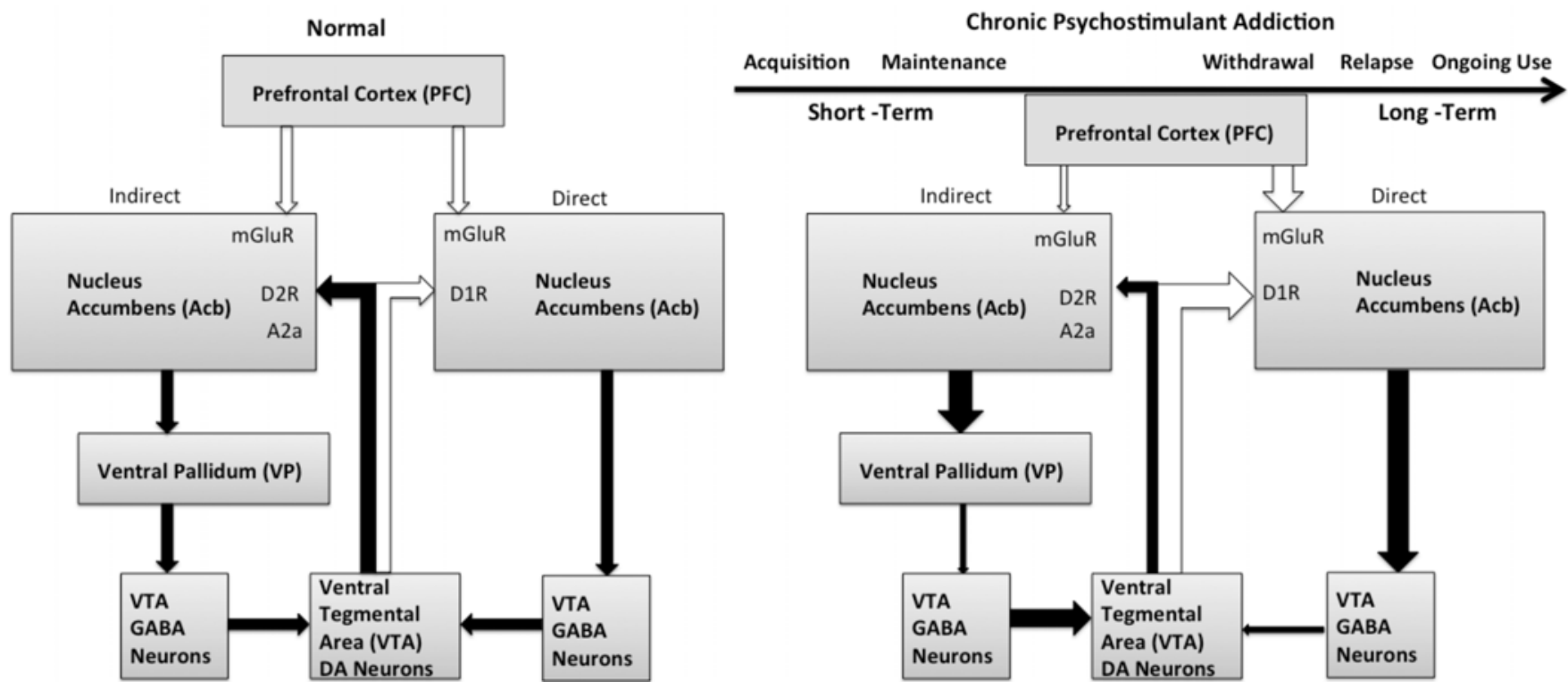

FIG. 7. Neuroadaptations in the PFC glutamatergic and VTA dopaminergic inputs to the direct and indirect pathways of the Acb that may occur in progressive, chronic psychostimulant addiction. White arrows = excitatory input (glutamate or dopamine); black arrows = inhibitory input (GABA or dopamine [DA]); arrow width depicts relative strength of input. A2a = adenosine receptor; D1R, $\mathrm{D} 2 \mathrm{R}=\mathrm{D}_{1 \mathrm{R}}, \mathrm{D}_{2 \mathrm{R}} ;$ mGluR = metabotropic glutamate receptor. 
amphetamine intake did not occur every day, and, when they did occur, reductions were on the order of approximately $40 \%$. If our acute findings were due to a DBS-induced increase in dopaminergic transmission in the AcbSh, then our inconsistent and moderate effect is likely a byproduct of our nonselective method of neuromodulation. Although Acb high-frequency DBS may activate midbrain dopaminergic projection terminals, it also activates local cholinergic and GABAergic interneurons, which serve to attenuate any enhanced terminal dopamine release. This is in contrast to selective optogenetic activation of the midbrain dopamine terminals only, which results in a more potent increase in accumbal dopamine transmission. ${ }^{43}$ The presumed mediocre improvement in dopamine transmission in the addicted brain following high-frequency DBS may not have been enough to sustain abstinence from methamphetamine in the long term. In addition, the DBSinduced changes in regional dopamine content that do occur may have far-reaching, and even contradictory, interactions with the multiple, and often opposing, pathways in the ventral striatum, which may explain the inter- and intraindividual variability of treatment responses that are noted not only here but in DBS for psychiatric disease at large. ${ }^{17}$

\section{Chronic Effects of DBS}

Remote effects of maintenance-phase AcbSh DBS were also observed in the form of reduced relapse 4-5 weeks later after a period of extinction and abstinence. Only 2 other studies have demonstrated a similar long-lasting effect of DBS on drug seeking and craving. Levy et al. delivered 30 minutes of PFC DBS to addicted rats every day for 10 days in the home cage directly following operant self-administration of cocaine. After the DBS therapy was stopped, rats exhibited a reduction in cue-induced cocaine seeking as well as in the motivation to obtain cocaine in both the short term (1 day after DBS) and the long term (9 days after DBS). ${ }^{36}$ Friedman and colleagues delivered DBS to cocaine-addicted rodents during the first 15 minutes of a 60-minute session in the operant chambers, which acutely decreased cocaine self-administration by more than $50 \%$ compared with a sham-stimulated group. DBS was then delivered in the same manner during 6 daily extinction sessions in which cocaine was not available. This paradigm reduced cocaine- and cue-induced reinstatement over the next 5 days even though DBS was not delivered on those days, suggesting a subacute effect of DBS therapy. ${ }^{18}$ Important contrasts between these studies and our work include the use of different brain targets, different stimulation parameters (e.g., Levy et al. used both low-frequency stimulation (LFS) and HFS while Friedman et al. used a combined pattern of alternating LFS and HFS), different timing of DBS therapy relative to drug use, different models of relapse, and of course, the use of cocaine versus methamphetamine. In the only other study in which the accumbens was targeted with repeated intermittent DBS, Hamilton et al. found that 30 minutes of daily Acb DBS for the first 14 days of abstinence did reduce cocaine-seeking behavior in the short term (1 day after DBS) but not in the long term (15 days after DBS), ${ }^{26}$ suggesting that stimulation effects dissipate over time when DBS is not continu- ous. This result is in agreement with results obtained by other investigators who noted that in rodents with a history of repeated cocaine exposure, a single session of 60-minute AcbSh HFS decreases cocaine-induced behavioral sensitization evidenced by enhanced locomotor response to cocaine, but this effect wears off by 4-24 hours after DBS. ${ }^{11}$

Despite the lack of supporting studies for long-term effects of limited-access DBS, there is a theoretical basis upon which to interpret our findings. As previously stated, repeated psychostimulant exposure enhances glutamatergic connections from the medial PFC to the $D_{1 R} M S N$ in the Acb as a type of drug-induced plasticity that manifests during withdrawal ${ }^{4,11,46}$ (Fig. 6). High-frequency AcbSh DBS, when delivered continuously during relapse testing, reduces drug-primed and cue-induced drug seeking in rats addicted to cocaine. ${ }^{24,58,59}$ This DBS effect is accompanied by increased neuronal activity in the medial PFC and is mimicked by intramedial PFC infusion of GABA agonists, ${ }^{59}$ suggesting that therapeutic DBS antidromically activates inhibitory GABA interneurons in the medial $\mathrm{PFC},{ }^{40}$ thereby decreasing the excessive glutamatergic input onto $\mathrm{D}_{1 \mathrm{R}}$ MSNs and interrupting the pathologically enhanced communication (Fig. 6). These acute DBS effects can become chronic if, instead of temporarily suppressing excessive excitatory input with traditional HFS, a novel DBS protocol of long-term depression is applied during early withdrawal that actually reverses drug-induced synaptic enhancement. ${ }^{11}$ Perhaps, in our study, DBS during the maintenance period of drug use is, a priori, interrupting the initial/ongoing process of long-term potentiation of glutamatergic afferents onto $\mathrm{D}_{1 \mathrm{R}} \mathrm{MSNs}$ via recurrent inhibition of the medial PFC as detailed above. It is exciting to speculate that neuromodulation in humans, even during a period of active drug use, could weaken the long-term potentiation that occurs between acute stimulus and pathological reward, thereby leading to decreased likelihood of relapse in the future.

\section{Weaknesses and Future Directions}

One obvious study weakness is that this investigation did not use a known human TMS stimulation protocol or brain target. However, the goal of this feasibility study was to first show that standard rodent DBS in a well-known reward-related target could affect drug behaviors when delivered at a different time and place than that of drug use. Follow-up studies are in progress by our group that address the outstanding issues related to TMS translation. Another weakness of this study is the fact that we failed to consistently decrease responding in the most aggressive methamphetamine responders (personal observation, 2013-2016). Unfortunately, these animals represent the target human population for neuromodulation, i.e., severely dependent drug abusers who have failed all other medical options. It is believed that the increased medial PFC-Acb $D_{1 \mathrm{R}}$ MSN glutamatergic tone that occurs in chronic psychostimulant administration is a generalized response to exposure that occurs in most animals during early or late withdrawal. ${ }^{14}$ This contrasts sharply with the increased medial PFC-Acb $\mathrm{D}_{2 \mathrm{R}}$ glutamatergic tone that occurs only in animals with a low motivation and compulsion for drug. ${ }^{5}$ While the $\mathrm{D}_{1 \mathrm{R}}$ 
MSN pathway may be involved in the rewarding, or hedonic, aspects of drug abuse, the $\mathrm{D}_{2 \mathrm{R}}$ MSN pathway may be involved in the regulation of compulsive drug using/ seeking. This "control" function of the indirect Abc pathway is supported by studies demonstrating its involvement in motivated drug self-administration ${ }^{5}$ and other unrestrained behaviors such as binge eating. ${ }^{25} \mathrm{~A}$ more selective stimulation protocol that could potentiate the $\mathrm{D}_{2 \mathrm{R}} \mathrm{MSN}$ pathway during ongoing drug use may increase control over drug compulsions and even be protective against the progression to addiction.

Much further work needs to be performed prior to translating these findings into actionable human therapy. The best region of the brain to stimulate for drug dependence remains to be determined; in fact, the best region of the Acb/ventral striatum remains unclear, with a recent paper suggesting that the lateral shell, not the traditional medioventral shell, ${ }^{13}$ may have the greatest VTA rewardrelated connections. ${ }^{4}$ The type of neuromodulation and the duration, parameters, and conditions of therapy are not well described, although noninvasive modalities are attractive options for the treatment of psychostimulant addiction given the catastrophic social situations that accompany many patients. It remains to be seen whether outpatient therapies such as TMS or transcranial direct current stimulation could be used as independent treatment strategies or simply as screening tools to better identify potential DBS responders.

\section{Conclusions}

This study demonstrates that it is feasible to alter methamphetamine intake and relapse using noncontinuous neuromodulation that is delivered at a different time and in a different environment than that of drug use. These effects did not alter the acute self-administration of food. Our findings have positive implications that noncontinuous electrical therapies delivered repeatedly in a sober environment could alter addiction behaviors in both the short and long term.

\section{Acknowledgments}

We would like to thank Sarah Harrold, Erika Arvidson, and Courtney Keller for technical suggestions and support. This study was supported by a 2014-2015 Neurosurgery Research and Education Foundation (NREF) award and a 2014 Grant-in-Aid Award from Louisiana State University Shreveport School of Medicine (to J.A.W.).

\section{References}

1. Ahmed SH, Koob GF: Transition from moderate to excessive drug intake: change in hedonic set point. Science 282:298300, 1998

2. Anderson SM, Bari AA, Pierce RC: Administration of the D1-like dopamine receptor antagonist SCH-23390 into the medial nucleus accumbens shell attenuates cocaine priminginduced reinstatement of drug-seeking behavior in rats. Psychopharmacology (Berl) 168:132-138, 2003

3. Batra V, Guerin GF, Goeders NE, Wilden JA: A general method for evaluating deep brain stimulation effects on intravenous methamphetamine self-administration. J Vis Exp 107:53266, 2016

4. Beier KT, Steinberg EE, DeLoach KE, Xie S, Miyamichi
K, Schwarz L, et al: Circuit architecture of VTA dopamine neurons revealed by systematic input-output mapping. Cell 162:622-634, 2015

5. Bock R, Shin JH, Kaplan AR, Dobi A, Markey E, Kramer PF, et al: Strengthening the accumbal indirect pathway promotes resilience to compulsive cocaine use. Nat Neurosci 16:632638, 2013

6. Brecht ML, Herbeck D: Time to relapse following treatment for methamphetamine use: a long-term perspective on patterns and predictors. Drug Alcohol Depend 139:18-25, 2014

7. Broom SL, Yamamoto BK: Effects of subchronic methamphetamine exposure on basal dopamine and stress-induced dopamine release in the nucleus accumbens shell of rats. Psychopharmacology (Berl) 181:467-476, 2005

8. Callaghan RC, Cunningham JK, Sykes J, Kish SJ: Increased risk of Parkinson's disease in individuals hospitalized with conditions related to the use of methamphetamine or other amphetamine-type drugs. Drug Alcohol Depend 120:35-40, 2012

9. Carcenac C, Favier M, Vachez Y, Lacombe E, Carnicella S, Savasta M, et al: Subthalamic deep brain stimulation differently alters striatal dopaminergic receptor levels in rats. Mov Disord 30:1739-1749, 2015

10. Courtney KE, Ray LA: Methamphetamine: an update on epidemiology, pharmacology, clinical phenomenology, and treatment literature. Drug Alcohol Depend 143:11-21, 2014

11. Creed M, Pascoli VJ, Lüscher C: Addiction therapy. Refining deep brain stimulation to emulate optogenetic treatment of synaptic pathology. Science 347:659-664, 2015

12. Cruickshank CC, Dyer KR: A review of the clinical pharmacology of methamphetamine. Addiction 104:1085-1099, 2009

13. Di Chiara G, Imperato A: Drugs abused by humans preferentially increase synaptic dopamine concentrations in the mesolimbic system of freely moving rats. Proc Natl Acad Sci U S A 85:5274-5278, 1988

14. Dobi A, Seabold GK, Christensen CH, Bock R, Alvarez VA: Cocaine-induced plasticity in the nucleus accumbens is cell specific and develops without prolonged withdrawal. J Neurosci 31:1895-1904, 2011

15. Feil J, Zangen A: Brain stimulation in the study and treatment of addiction. Neurosci Biobehav Rev 34:559-574, 2010

16. Figee M, de Koning P, Klaassen S, Vulink N, Mantione M, van den Munckhof P, et al: Deep brain stimulation induces striatal dopamine release in obsessive-compulsive disorder. Biol Psychiatry 75:647-652, 2014

17. Fitzgerald PB, Segrave RA: Deep brain stimulation in mental health: Review of evidence for clinical efficacy. Aust $\mathbf{N} \mathbf{Z} \mathbf{J}$ Psychiatry 49:979-993, 2015

18. Friedman A, Lax E, Dikshtein Y, Abraham L, Flaumenhaft Y, Sudai E, et al: Electrical stimulation of the lateral habenula produces enduring inhibitory effect on cocaine seeking behavior. Neuropharmacology 59:452-459, 2010

19. Fuchs RA, Ramirez DR, Bell GH: Nucleus accumbens shell and core involvement in drug context-induced reinstatement of cocaine seeking in rats. Psychopharmacology (Berl) 200:545-556, 2008

20. Grall-Bronnec M, Sauvaget A: The use of repetitive transcranial magnetic stimulation for modulating craving and addictive behaviours: a critical literature review of efficacy, technical and methodological considerations. Neurosci Biobehav Rev 47:592-613, 2014

21. Granado N, Ares-Santos S, Moratalla R: Methamphetamine and Parkinson's disease. Parkinsons Dis 2013:308052, 2013

22. Groman SM, Morales AM, Lee B, London ED, Jentsch JD: Methamphetamine-induced increases in putamen gray matter associate with inhibitory control. Psychopharmacology (Berl) 229:527-538, 2013 
23. Grueter BA, Rothwell PE, Malenka RC: Integrating synaptic plasticity and striatal circuit function in addiction. Curr Opin Neurobiol 22:545-551, 2012

24. Guercio LA, Schmidt HD, Pierce RC: Deep brain stimulation of the nucleus accumbens shell attenuates cue-induced reinstatement of both cocaine and sucrose seeking in rats. Behav Brain Res 281:125-130, 2015

25. Halpern CH, Tekriwal A, Santollo J, Keating JG, Wolf JA, Daniels D, et al: Amelioration of binge eating by nucleus accumbens shell deep brain stimulation in mice involves D2 receptor modulation. J Neurosci 33:7122-7129, 2013

26. Hamilton J, Lee J, Canales JJ: Chronic unilateral stimulation of the nucleus accumbens at high or low frequencies attenuates relapse to cocaine seeking in an animal model. Brain Stimulat 8:57-63, 2015

27. Hauser SR, Wilden JA, Deehan GA Jr, McBride WJ, Rodd ZA: Cocaine influences alcohol-seeking behavior and relapse drinking in alcohol-preferring (P) rats. Alcohol Clin Exp Res 38:2678-2686, 2014

28. Henning J, Koczan D, Glass A, Karopka T, Pahnke J, Rolfs A, et al: Deep brain stimulation in a rat model modulates TH, CaMKIIa and Homerl gene expression. Eur J Neurosci 25:239-250, 2007

29. Jedynak J, Hearing M, Ingebretson A, Ebner SR, Kelly M, Fischer RA, et al: Cocaine and amphetamine induce overlapping but distinct patterns of AMPAR plasticity in nucleus accumbens medium spiny neurons. Neuropsychopharmacology 41:464-476, 2016

30. Kamei H, Nagai T, Nakano H, Togan Y, Takayanagi M, Takahashi K, et al: Repeated methamphetamine treatment impairs recognition memory through a failure of novelty-induced ERK1/2 activation in the prefrontal cortex of mice. Biol Psychiatry 59:75-84, 2006

31. Katner SN, Oster SM, Ding ZM, Deehan GA Jr, Toalston JE, Hauser SR, et al: Alcohol-preferring $(\mathrm{P})$ rats are more sensitive than Wistar rats to the reinforcing effects of cocaine self-administered directly into the nucleus accumbens shell. Pharmacol Biochem Behav 99:688-695, 2011

32. Koob GF, Volkow ND: Neurocircuitry of addiction. Neuropsychopharmacology 35:217-238, 2010

33. Kousik SM, Carvey PM, Napier TC: Methamphetamine selfadministration results in persistent dopaminergic pathology: implications for Parkinson's disease risk and reward-seeking. Eur J Neurosci 40:2707-2714, 2014

34. Laćan G, Hadamitzky M, Kuczenski R, Melega WP: Alterations in the striatal dopamine system during intravenous methamphetamine exposure: effects of contingent and noncontingent administration. Synapse 67:476-488, 2013

35. Le Cozannet R, Markou A, Kuczenski R: Extended-access, but not limited-access, methamphetamine self-administration induces behavioral and nucleus accumbens dopamine response changes in rats. Eur J Neurosci 38:3487-3495, 2013

36. Levy D, Shabat-Simon M, Shalev U, Barnea-Ygael N, Cooper A, Zangen A: Repeated electrical stimulation of reward-related brain regions affects cocaine but not "natural" reinforcement. J Neurosci 27:14179-14189, 2007

37. Lobo MK, Covington HE III, Chaudhury D, Friedman AK, Sun H, Damez-Werno D, et al: Cell type-specific loss of BDNF signaling mimics optogenetic control of cocaine reward. Science 330:385-390, 2010

38. Lominac KD, McKenna CL, Schwartz LM, Ruiz PN, Wroten MG, Miller BW, et al: Mesocorticolimbic monoamine correlates of methamphetamine sensitization and motivation. Front Syst Neurosci 8:70, 2014

39. Marshall BD, Werb D: Health outcomes associated with methamphetamine use among young people: a systematic review. Addiction 105:991-1002, 2010

40. McCracken CB, Grace AA: Nucleus accumbens deep brain stimulation produces region-specific alterations in local field potential oscillations and evoked responses in vivo. J Neurosci 29:5354-5363, 2009

41. McKetin R, Lubman DI, Najman JM, Dawe S, Butterworth P, Baker AL: Does methamphetamine use increase violent behaviour? Evidence from a prospective longitudinal study. Addiction 109:798-806, 2014

42. Meissner W, Harnack D, Paul G, Reum T, Sohr R, Morgenstern R, et al: Deep brain stimulation of subthalamic neurons increases striatal dopamine metabolism and induces contralateral circling in freely moving 6-hydroxydopamine-lesioned rats. Neurosci Lett 328:105-108, 2002

43. Melchior JR, Ferris MJ, Stuber GD, Riddle DR, Jones SR: Optogenetic versus electrical stimulation of dopamine terminals in the nucleus accumbens reveals local modulation of presynaptic release. J Neurochem 134:833-844, 2015

44. Panenka WJ, Procyshyn RM, Lecomte T, MacEwan GW, Flynn SW, Honer WG, et al: Methamphetamine use: a comprehensive review of molecular, preclinical and clinical findings. Drug Alcohol Depend 129:167-179, 2013

45. Parsegian A, See RE: Dysregulation of dopamine and glutamate release in the prefrontal cortex and nucleus accumbens following methamphetamine self-administration and during reinstatement in rats. Neuropsychopharmacology 39:811822,2014

46. Pascoli V, Turiault M, Lüscher C: Reversal of cocaine-evoked synaptic potentiation resets drug-induced adaptive behaviour. Nature 481:71-75, 2011

47. Paxinos G, Watson CR, Emson PC: AChE-stained horizontal sections of the rat brain in stereotaxic coordinates. J Neurosci Methods 3:129-149, 1980

48. Pontieri FE, Tanda G, Di Chiara G: Intravenous cocaine, morphine, and amphetamine preferentially increase extracellular dopamine in the "shell" as compared with the "core" of the rat nucleus accumbens. Proc Natl Acad Sci U S A 92:12304-12308, 1995

49. Rocha A, Kalivas PW: Role of the prefrontal cortex and nucleus accumbens in reinstating methamphetamine seeking. Eur J Neurosci 31:903-909, 2010

50. Rodd ZA, Bell RL, Sable HJ, Murphy JM, McBride WJ: Recent advances in animal models of alcohol craving and relapse. Pharmacol Biochem Behav 79:439-450, 2004

51. Rogers JL, De Santis S, See RE: Extended methamphetamine self-administration enhances reinstatement of drug seeking and impairs novel object recognition in rats. Psychopharmacology (Berl) 199:615-624, 2008

52. Shepard JD, Chuang DT, Shaham Y, Morales M: Effect of methamphetamine self-administration on tyrosine hydroxylase and dopamine transporter levels in mesolimbic and nigrostriatal dopamine pathways of the rat. Psychopharmacology (Berl) 185:505-513, 2006

53. Smith RJ, Lobo MK, Spencer S, Kalivas PW: Cocaineinduced adaptations in D1 and D2 accumbens projection neurons (a dichotomy not necessarily synonymous with direct and indirect pathways). Curr Opin Neurobiol 23:546-552, 2013

54. Stefanik MT, Moussawi K, Kupchik YM, Smith KC, Miller RL, Huff ML, et al: Optogenetic inhibition of cocaine seeking in rats. Addict Biol 18:50-53, 2013

55. Steketee JD, Sorg BA, Kalivas PW: The role of the nucleus accumbens in sensitization to drugs of abuse. Prog Neuropsychopharmacol Biol Psychiatry 16:237-246, 1992

56. Terraneo A, Leggio L, Saladini M, Ermani M, Bonci A, Gallimberti L: Transcranial magnetic stimulation of dorsolateral prefrontal cortex reduces cocaine use: A pilot study. Eur Neuropsychopharmacol 26:37-44, 2016

57. van Dijk A, Klompmakers AA, Feenstra MG, Denys D: Deep brain stimulation of the accumbens increases dopamine, serotonin, and noradrenaline in the prefrontal cortex. J Neurochem 123:897-903, 2012 
58. Vassoler FM, Schmidt HD, Gerard ME, Famous KR, Ciraulo DA, Kornetsky C, et al: Deep brain stimulation of the nucleus accumbens shell attenuates cocaine priming-induced reinstatement of drug seeking in rats. J Neurosci 28:8735-8739, 2008

59. Vassoler FM, White SL, Hopkins TJ, Guercio LA, Espallergues J, Berton O, et al: Deep brain stimulation of the nucleus accumbens shell attenuates cocaine reinstatement through local and antidromic activation. J Neurosci 33:14446-14454, 2013

60. Volkow ND, Chang L, Wang GJ, Fowler JS, Franceschi D, Sedler M, et al: Loss of dopamine transporters in methamphetamine abusers recovers with protracted abstinence. J Neurosci 21:9414-9418, 2001

61. Volkow ND, Chang L, Wang GJ, Fowler JS, Leonido-Yee M, Franceschi D, et al: Association of dopamine transporter reduction with psychomotor impairment in methamphetamine abusers. Am J Psychiatry 158:377-382, 2001

62. Wang L, Shen M, Yu Y, Tao Y, Zheng P, Wang F, et al: Optogenetic activation of GABAergic neurons in the nucleus accumbens decreases the activity of the ventral pallidum and the expression of cocaine-context-associated memory. Int J Neuropsychopharmacol 17:753-763, 2014

63. Wilden JA, Qing KY, Hauser SR, McBride WJ, Irazoqui PP, Rodd ZA: Reduced ethanol consumption by alcohol-preferring $(\mathrm{P})$ rats following pharmacological silencing and deep brain stimulation of the nucleus accumbens shell. J Neurosurg 120:997-1005, 2014

64. Yamamoto T, Uchiyama T, Higuchi Y, Asahina M, Hirano S, Yamanaka Y, et al: Subthalamic nucleus deep brain stimulation modulate catecholamine levels with significant relations to clinical outcome after surgery in patients with Parkinson's disease. PLoS One 10:e0138462, 2015

\section{Disclosures}

The authors report no conflict of interest concerning the materials or methods used in this study or the findings specified in this paper.

\section{Author Contributions}

Conception and design: Wilden, Batra, Guerin, Goeders. Acquisition of data: Batra, Tran, Caputo, Guerin. Analysis and interpretation of data: Wilden. Drafting the article: Wilden. Critically revising the article: Wilden, Batra, Tran, Caputo. Reviewed submitted version of manuscript: Wilden. Approved the final version of the manuscript on behalf of all authors: Wilden. Statistical analysis: Wilden, Batra. Administrative/technical/material support: Guerin, Goeders. Study supervision: Wilden, Guerin, Goeders.

\section{Supplemental Information}

\section{Previous Presentations}

Portions of this work were presented in poster format at the Society for Neurosciences Annual Meeting in Chicago, Illinois, in October 2015

\section{Current Affiliations}

Dr. Wilden: Tri-State Neurosurgery, Shreveport, LA.

\section{Correspondence}

Jessica Wilden, Tri-State Neurosurgery, 2551 Greenwood Rd., Ste. 320, Shreveport, LA 71103. email: jwild2@1suhsc.edu. 